Article Type: 3 Original Article - Australia, Japan, SE Asia

\title{
A LONGITUDINAL STUDY OF THYROID AUTOANTIBODIES IN PREGNANCY: THE IMPORTANCE OF TEST TIMING.
}

\author{
Abbreviated title: thyroid autoantibodies in pregnancy \\ Elif I Ekinci ${ }^{1,2,3}$ MBBS, FRACP, PhD, Wei-Ling Chiu*1 MBChB, BSci, Zhong X Lu ${ }^{4,5}$ MBBS, \\ FRCPA, PhD, Ken Sikaris ${ }^{4}$ MBBS, FRCPA, Leonid Churilov ${ }^{6,7}$ BSc(Hons), PhD, Intissar Bittar ${ }^{8}$ \\ BSci, Que Lam $^{8}$ MBBS, FRCPA, Nick Crinis ${ }^{8}$ BSci, Christine A Houlihan ${ }^{1,9}$ MBBS, FRACP, MD. \\ ${ }^{1}$ Department of Endocrinology and ${ }^{8}$ Department of Biochemistry, Austin Health, Melbourne; \\ ${ }^{2}$ Department of Medicine, University of Melbourne, Melbourne; ${ }^{3}$ Menzies School of Health Research, \\ Darwin, Australia; ${ }^{4}$ Department of Chemical Pathology, Melbourne Pathology, Melbourne; \\ ${ }^{5}$ Department of Medicine, Monash University, Melbourne; ${ }^{6}$ Florey Institute of Neuroscience and \\ Mental Health, Melbourne; ${ }^{7}$ School of Mathematical and Geospatial Sciences, RMIT University, \\ Melbourne; ${ }^{9}$ Mercy Hospital for Women, Melbourne. \\ *Equal first author contribution
}

Correspondence: Dr Christine A Houlihan, Perinatal Unit, Level 3, Mercy Hospital for Women, 163 Studley Rd, Heidelberg, Victoria 3084, Australia.

Email: CHoulihan@mercy.com.au_Ph : +61394965000Ｆax : +61 398550715

Key Terms: postpartum, Hashimoto's, thyroid, autoimmunity, and pregnancy

Acknowledgments: We would like to acknowledge Ms Vasantha Ragunathan for assisting with patient recruitment; Dr Sujiva Ratnaike for her intellectual contribution and comments on the manuscript; Melbourne Pathology for assisting in home visits for the collection of the postpartum bloods and for performing all the laboratory analyses; and Roche Diagnostics for providing all the reagent kits for this study. EIE is supported by an NHMRC Early Career Fellowship This article has been accepted for publication and undergone full peer review but has not been through the copyediting, typesetting, pagination and proofreading process, which may lead to differences between this version and the Version of Record. Please cite this article as doi: $10.1111 /$ cen.12571 This article is protected by copyright. All rights reserved. 
(APP\#APP1054312). The Florey Institute of Neuroscience and Mental Health acknowledges the support from the Victorian Government's Operational Infrastructure Support Grant.

Conflict of interest and financial disclosure: Nothing to declare.

Grants supporting the project: Medical Research Foundation for Women and Babies

Ethics approval: The ethics committee of Mercy Hospital for Women approved this study (MHAC HREC Project Reference Number: R06/R07)

\begin{abstract}
Objective: Thyroid-peroxidase antibodies (TPOAb) and thyroglobulin antibodies (TGAb) are frequently measured to investigate thyroid dysfunction in pregnancy. Despite the recognized fall of these autoantibodies in pregnancy, there is limited guidance on the timing of such testing. We assessed optimal test timing of TPOAb/TGAb for detection of Hashimoto's thyroiditis and postpartum thyroid dysfunction (PPTD).
\end{abstract}

Design: Prospective longitudinal study with recruitment in Trimester-1.

Patients: Healthy women $\leq 13$ weeks gestation from Mercy Hospital for Women, a tertiary obstetric hospital in Melbourne.

Measurements: Serum TPOAb, TGAb, TSH and fT4 were measured at Trimester-1(T1), Trimester2(T2), Trimester-3(T3), and postpartum(PP) in each participant. Postpartum thyroid dysfunction (PPTD) was defined if TSH deviated from the assay's non-pregnant reference interval. Longitudinal random-effect logistic regression was used to investigate the association between time and positive/negative thyroid autoantibody status.

Results: Samples from 140 women at T1(12.0:10.3-13.0) (median:IQR weeks gestation); 95 at $\mathrm{T} 2(24.3: 23.0-25.9), 79$ at $\mathrm{T} 3(35.9: 34.8-36.7)$ and 83 at $\mathrm{PP}(12.4: 10.8-14.6$ weeks postpartum) were

This article is protected by copyright. All rights reserved. 
attained. At T1, 13(9\%) and 15(11\%) women had positive TPOAb and TGAb, respectively. The odds of having a positive TPOAb was 96\% lower at T2(OR=0.04 (95\%CI:0.02-0.8; $p=0.03)$ ) and 97\% lower at $\mathrm{T} 3(\mathrm{OR}=0.03(95 \% \mathrm{CI}: 0.001-0.6 ; p=0.02))$ than at $\mathrm{T} 1$. Similarly, the odds of having a positive TGAb was $99.4 \%$ lower $(\mathrm{OR}=0.006(95 \% \mathrm{CI}: 0-0.3 ; p=0.01))$ at $\mathrm{T} 2$, and $99.5 \%$ lower $(\mathrm{OR}=0.005(95 \% \mathrm{CI}: 0-0.4 ; p=0.02))$ at $\mathrm{T} 3$ than at $\mathrm{T} 1$. The ROC analysis diagnostic ORs for a positive $\mathrm{TPOAb}$ and/or TGAb to predict PPTD were 7.8(95\%CI:2.2-27.6), 1.2(95\%CI:0-8.9), 2.0(95\%CI:016.8), and 12.2(95\%CI:3.3-44.9) at T1, T2, T3 and postpartum, respectively.

Conclusions: A significant proportion of pregnant women lose their thyroid autoantibody positivity after $\mathrm{T} 1$. The gestation dependent loss of TPOAb/TGAb positivity and reduction in diagnostic accuracy for predicting PPTD limits the value of testing at T2 and T3.

\section{INTRODUCTION}

The commonest cause of hypothyroidism is Hashimoto's thyroiditis in iodine-replete countries (1). Thyroid autoantibodies are frequently measured, often for the first time, in women found to have overt or subclinical hypothyroidism in pregnancy to determine if Hashimoto's thyroiditis is the likely underlying pathology (1). The prevalence of thyroid peroxidase antibodies (TPOAb) and thyroglobulin antibodies (TGAb) has been reported to be $15 \%$ and $14 \%$, respectively, in a large cohort of disease-free women in the NHANES III study (2). Among women of reproductive age, the prevalence was $10-13 \%$ for TPOAb and 9-14\% for TGAb (2). In pregnancy, up to $20 \%$ of pregnant women in the first trimester have tested positive for either or both antibodies (3). The presence of TPOAb in particular has been associated with reduced thyroid functional reserve with possible development of hypothyroidism during gestation (4). Detection of TPOAb in early pregnancy also imparts a 50\% risk for the development of postpartum thyroid dysfunction (5).

There exists a strong interrelationship between thyroid function and pregnancy. In women with limited thyroid reserve, thyroid dysfunction may develop in the setting of increased thyroxine requirements in pregnancy $(4,6)$. Conversely, maternal hypothyroidism, both overt and subclinical, can cause adverse pregnancy $(7-9)$ and fetal outcomes $(10,11)$. Previous studies have demonstrated

This article is protected by copyright. All rights reserved. 
decreases in thyroid autoantibody levels, with advancing gestation during pregnancy $(4,12-14)$. Some have shown this to occur by parturition (4) while others have shown it to be evident earlier, occurring between 12 and 28 weeks gestation (14). The interpretation of thyroid autoantibody tests, therefore, needs to take place in the context of known fluctuations of antibody levels in pregnancy. Although clinical guidelines do not recommend universal screening of thyroid autoantibodies in pregnancy (15, 16), testing of thyroid autoantibodies is frequently performed in pregnant women identified with overt or subclinical hypothyroidism. Furthermore, the American Thyroid Association guideline uses TPOAb status as a determinant for its recommendations for treatment of subclinical hypothyroidism in pregnancy $(15,16)$. Thus, in the current prospective longitudinal study, we sought to examine the effect that the positive/negative status of thyroid autoantibodies, measured longitudinally in each trimester and at postpartum, would have on the ability to (i) diagnose Hashimoto's thyroiditis and (ii) the three trimester measures to be used as a screening tool for the occurrence of postpartum thyroid dysfunction.

\section{MATERIALS AND METHODS}

The present study population is a cohort of healthy pregnant women, initially recruited for a longitudinal study to determine trimester and method specific reference intervals for thyroid function tests (17). In the current analysis, we present data on the longitudinal changes of TPOAb and TGAb throughout each trimester of pregnancy and postpartum. Women presenting to Mercy Hospital for Women, a tertiary obstetric hospital in Melbourne, at or prior to 13 weeks gestation were recruited between May 2006 and February 2011(17). One hundred and fifty-four healthy women without any history of thyroid disease were recruited.

Exclusion criteria included past history of thyroid disease, thyroid hormone replacement therapy, type 1 diabetes mellitus, past history of intravenous drug abuse or presence of major systemic illness. Women with twin pregnancy and miscarriage prior to 20 weeks in the current pregnancy were also excluded from the analysis.

As reported in our previous study (17), three women were commenced on thyroxine when their thyroid stimulating hormone (TSH) at $\mathrm{T} 1$ was found to be $\geq 2.5 \mathrm{mIU} / \mathrm{L}$ as recommended by

This article is protected by copyright. All rights reserved. 
international guidelines (15). These women all had negative TPOAb and TGAb. Only the initial (prethyroxine therapy) blood samples at $\mathrm{T} 1$ of these women were included in the statistical analysis of thyroid function but all samples were included for thyroid autoantibody analysis.

\section{Procedures}

Blood samples (20 mL each) were taken at 9-13 weeks, 22-26 weeks, and 35-39 weeks of gestation to represent Trimester-1 (T1), Trimester-2 (T2) and Trimester-3 (T3), and 8-12 weeks postpartum (PP). Late postpartum samples, up to 20 weeks postpartum, were not excluded. Measurements in T2, T3 and PP were on the same group of patients (less missing samples) tested in T1. Urine specimens were collected from each woman at T1. The Human Research Ethics Committee at MHW approved the study and written informed consent was obtained from each study participant.

\section{Assay Methods}

Serum TPOAb, TGAb, TSH and free thyroxine (fT4) concentrations were measured at all time-points, using Roche e602 analysers (Roche Diagnostics, Mannheim, Germany) at Melbourne Pathology. Urine iodine concentration was measured using Inductively Coupled Plasma Mass Spectrometry (ICPMS) method with the Agilent $7500 \mathrm{CE}$ instrument with an octopole reaction cell. The limit of detection for TSH was $0.01 \mathrm{mIU} / \mathrm{L}$. The inter-assay coefficients of variation $(\mathrm{CV})$ were $7.2 \%$ at 0.06 $\mathrm{mIU} / \mathrm{L}$ and $2.5 \%$ at $\geq 1.0 \mathrm{mIU} / \mathrm{L}$ for TSH; $2.9 \%$ for fT4 at $11.0 \mathrm{pmol} / \mathrm{L} ; 9.7 \%$ for TPOAb at $35 \mathrm{IU} / \mathrm{mL}$ and $5.5 \%$ at $\geq 120 \mathrm{IU} / \mathrm{mL} ; 9.5 \%$ for $\mathrm{TGAb}$ at $60 \mathrm{IU} / \mathrm{mL}$ and $4.0 \%$ at $\geq 450 \mathrm{IU} / \mathrm{mL}$; and $2.3 \%$ for urine iodine concentration at $92 \mathrm{ug} / \mathrm{L}$. The laboratory determined 97.5 th percentile for the gestationspecific, assay-specific and population-specific TSH reference intervals (TPOAb negative women) were 2.6, 4.8 and 4.8mIU/L at T1, T2, and T3, respectively.

\section{Outcome measures}

Thyroid antibody status was dichotomized as positive versus negative; values of TPOAb $<35 \mathrm{IU} / \mathrm{mL}$ and TGAb $<115 \mathrm{IU} / \mathrm{mL}$ were defined as being negative. "Combined positive" was defined as TPOAb $\geq 35 \mathrm{IU} / \mathrm{mL}$ and/or TGAb $\geq 115 \mathrm{IU} / \mathrm{mL}$. Postpartum thyroid dysfunction (PPTD) was defined based on biochemical grounds in this study. Women were classified as having PPTD if their TSH deviated

This article is protected by copyright. All rights reserved. 
from the non-pregnant reference interval of $0.5-5.0 \mathrm{mIU} / \mathrm{L}$ for the Roche assay in the postpartum period.

\section{Statistical Analysis}

The statistical analysis was performed using STATA 13IC statistical software (College Station, TX). TPOAb and TGAb levels across time-points have been presented as scatterplots with 25 th, median and 75 th percentile values. Due to the longitudinal nature of the measurement data, a multi-level random-effect logistic regression model was used to investigate the association between the time (measured at four time-points, i.e. three trimesters and postpartum) and the outcome measures (thyroid antibody status dichotomized as positive versus negative), adjusted for age and TSH. The sensitivity, specificity, positive and negative predictive values, as well as the diagnostic Odds Ratios were estimated using the "combined positive" antibody status as a screening test for PPTD and reported with corresponding 95\% confidence intervals (CI).

\section{RESULTS}

Women with miscarriage prior to 20 weeks $(n=7)$, and twin pregnancy $(n=2)$ were excluded from the analysis. Of the seven women with miscarriage, one had positive TGAb and none had positive TPOAb in T1. Data were available from 140 women at T1 (12.0: 10.3-13.0) (median: IQR weeks gestation); 95 at T2 (24.3: 23.0-25.9), 79 at T3 (35.9: 34.8-36.7) and 83 at PP (12.4: 10.8-14.6 weeks postpartum).

\section{Baseline Characteristics}

The baseline characteristics of the study population are summarized in Table 1. The median (IQR: $25^{\text {th }}-75^{\text {th }}$ percentiles) urine iodine concentration of the population was $137.8(83.0-232.1) \mu \mathrm{g} / \mathrm{L}$. No statistically significant association between baseline urinary iodine concentration and TSH levels at any time-points were detected.

This article is protected by copyright. All rights reserved. 
Twenty-two (16\%) women tested positive for TPOAb and/or TGAb at T1: six were positive for both autoantibodies, seven positive for TPOAb alone and nine positive for TGAb alone.

Compared to the TPOAb negative group at T1, the TPOAb positive group was older (median: 34.6 (IQR: 29.4, 39.4) versus 31.3 (IQR: 28.3, 34.5) years, Wilcoxon-Mann-Whitney $p=0.04$ ) with higher TSH (median: 1.48 (IQR: 1.27, 1.89) versus 0.88 (IQR: 0.53, 1.5) mIU/L, Wilcoxon-Mann-Whitney $p=0.002$ ). No statistically significant differences in gravida or parity according to antibody status for TPOAb and TGAb at baseline were detected.

\section{Thyroid autoantibodies}

The distribution of thyroid autoantibodies across each trimester and postpartum, for the entire study population, are shown in Figure 1.

The longitudinal changes of thyroid autoantibody levels in individuals with positive TPOAb and TGAb at T1 across pregnancy and postpartum are shown in Figure 2. In women with positive TPOAb at T1 (Figure 2a), a progressive decline in autoantibody levels in pregnancy followed by an increase at postpartum was observed; at T2 and T3, 56\% (5/9) (95\%CI: 21\%-86\%) and 43\% (3/7) (95\%CI: 10\%-82\%) of women, respectively, remained positive for TPOAb, and at PP, all (9/9) (95\%CI: 66\%-100\%) women developed TPOAb positivity again. A similar pattern of change in TGAb levels over time was also seen in women with positive TGAb at T1 (Figure 2b); at T2 and T3, $36 \%$ (4/11) (95\%CI: 11\%-63\%) and 33\% (3/9) (95\%CI: 7\%-70\%), respectively, remained positive for TGAb and 92\% (11/12) (95\%CI: 62\%-100\%) were positive again at PP.

Compared to $\mathrm{T} 1$, the odds of having a positive TPOAb was $96 \%$ lower at $\mathrm{T} 2(\mathrm{OR}=0.04(95 \%$ CI: $0.02-0.8 ; p=0.03$ ), and 97\% lower at T3 (OR=0.03 (95\% CI: 0.001-0.6; $p=0.02)$. Similarly, compared to T1, the odds of having a positive TGAb was $99.4 \%$ lower at T2 (OR=0.006 (95\% CI: 0$0.3 ; p=0.01$ ), and $99.5 \%$ lower at T3 (OR=0.005 (95\%CI: $0-0.4 ; p=0.02)$. No significant differences between $\mathrm{T} 1$ and postpartum were observed for either autoantibody (Table 2).

This article is protected by copyright. All rights reserved. 
In women with negative TPOAb and TGAb at $\mathrm{T} 1$, the changes in thyroid autoantibody levels across pregnancy and postpartum were minimal. One subject who was negative at $\mathrm{T} 1 \mathrm{for}$ TPOAb became positive postpartum, while all others remained negative throughout. All women who were negative at $\mathrm{T} 1$ for TGAb remained negative throughout pregnancy and postpartum.

\section{Thyroid function}

At $\mathrm{T} 2$ or $\mathrm{T} 3$, there were no significant differences in thyroid function between autoantibody positive and negative groups for either TPOAb or TGAb, respectively. For women with "combined positive" antibodies at T1, none had an increase in TSH at T2 or T3 above the 97.5 th percentile for the gestation-specific, assay-specific and population-specific reference range. At PP, after exclusion of the 13 subjects with PPTD (see below) no statistically significant differences in TSH and fT4, according to antibody status, were detected.

\section{PPTD}

At PP, 13 of 83 (16\%) (95\%CI: 9\%-25\%) women had biochemical evidence of PPTD: 10 had TSH $<0.5 \mathrm{mIU} / \mathrm{L}$ (four of whom had TSH $<0.02 \mathrm{mIU} / \mathrm{L}$ ) and three had TSH $>5.0 \mathrm{mIU} / \mathrm{L}$.

The prevalence of PPTD was found to be higher in women with positive TPOAb and/or TGAb at T1 than those with negative thyroid antibodies at T1 (32\% (95\% CI: $13.9 \%-54.8 \%)$ versus $5.1 \%(95 \%$

CI: $1.8 \%-10.7 \%)$.

The ability to predict the occurrence of PPTD using the "combined positive" antibody status as a screening test for PPTD is shown in Table 3. The table contains estimations of the sensitivity, specificity, positive and negative predictive values, as well as the diagnostic Odds Ratios (ORs). The ROC analysis showed that the ratio of the odds of "combined positive" antibodies in patients with PPTD, relative to the odds of "combined positive" antibodies in patients without PPTD (diagnostic ORs) to be 7.8 (95\%CI:2.2-27.6), 1.2 (95\%CI:0-8.9), 2.0 (95\%CI:0-16.8), and 12.2 (95\%CI:3.3-44.9) at $\mathrm{T} 1, \mathrm{~T} 2, \mathrm{~T} 3$ and postpartum, respectively.

This article is protected by copyright. All rights reserved. 


\section{DISCUSSION}

In this study, we determined the longitudinal changes of TPOAb and TGAb throughout pregnancy and postpartum, and found that thyroid autoantibody positivity decreased between 12 and 25 weeks gestation, remained lowered throughout the third trimester and increased again by 12 weeks postpartum. The decline in rates of thyroid autoantibody positivity in pregnancy is considered a manifestation of maternal immune tolerance, and the return of thyroid autoimmunity postpartum due to cessation of pregnancy-mediated immunosuppression $(18,19)$.

The prevalence of thyroid autoantibodies in this present cohort, an iodine insufficient urban population, was $16 \%$. A strength of the present study is that, in contrast to previous studies, it provides data from all three trimesters and postpartum $(4,13)$. Similar to other longitudinal studies of thyroid autoantibodies in pregnancy, the present study showed a significant decline in rates of TPOAb and TGAb positivity between T1 and T3 $(4,12-14,20)$, and more specifically to be evident by 24 weeks gestation. A large prospective study by Glinoer et al (4) in 87 thyroid autoantibody positive patients showed $60 \%$ reduction in TPOAb levels and a decrease in TPO positivity rate such that less than $54 \%$ of those positive at $\mathrm{T} 1$, remained positive at parturition. A smaller prospective study (12), where patients were followed up to six weeks postpartum, showed a similar decrease in autoantibody positivity in pregnancy with rebound of TPOAb but not TGAb at postpartum; a difference that may be related to the small sample size or the earlier postpartum testing at 6-weeks in that study. In addition, our study showed that the majority of women with negative thyroid autoantibodies at $\mathrm{T} 1$ remained negative throughout the rest of the pregnancy and postpartum. The stability of TPOAb and TGAb levels over a 6-month period in a non- pregnant population has previously been demonstrated (21) and therefore supports the assumption that changes in thyroid autoantibody levels can be attributed to the pregnancy.

In the present study, evidence of impaired thyroid reserve was found at T1, with the TPOAb positive group having significantly higher TSH than the TPOAb negative group. The observation of an impaired thyroid response to pregnancy in thyroid autoantibody positive subjects was first noted by Glineor et al two decades ago (4). In that study of women with asymptomatic autoimmune thyroid

This article is protected by copyright. All rights reserved. 
disease who were euthyroid in early pregnancy, the TSH levels remained significantly higher at all time-points during pregnancy compared to those in the antibody negative group (4). Similarly, in a randomised controlled trial of thyroxine replacement in pregnancy, the untreated 'euthyroid' TPOAb positive women (compared to negative TPOAb women), demonstrated higher TSH levels (7).

A previous study has shown that $16 \%$ of euthyroid women with positive TPOAb or TGAb in the first trimester had a TSH exceeding $4.0 \mathrm{mIU} / \mathrm{L}$ by the third trimester (4). In the present study, of the 22 euthyroid women with positive TPOAb and/or TGAb at T1, none had an increase in TSH above the 97.5th percentile for the gestation-specific, assay-specific and population-specific reference range. Possible reasons for the differences between the two studies could relate to the different assay methods used, differences in iodine intake, and the use of non-trimester-specific ranges in the

\section{Glinoer study.}

There is a large variation in the reported prevalence of postpartum thyroiditis, ranging from $1.1-21.1 \%$ (22). In iodine sufficient areas, there is an estimated prevalence of 5-7\% (22). A previous Australian study of randomly selected postpartum Caucasian women aged 20-45 years who had thyroid testing performed at 4.5-5.5 months postpartum reported an 11.5\% prevalence of PPTD, defined as TSH or fT4 outside the adult reference range (23). Some variability between studies may relate to different postpartum testing times, methodological considerations, environmental and genetic factors in different populations (22). Consistent with previous studies $(20,23)$, the prevalence of PPTD was found to be much higher in women with positive TPOAb and/or TGAb at T1 than those with negative thyroid antibodies. The 54\% PPTD prevalence in women with positive TPOAb at T1, acknowledging wide confidence intervals, compares with previous reports of between 33-66\% (13, $15,24)$.

In the present study, the sensitivity of a combined positive antibody result to predict PPTD, more than halved, when these antibodies were measured at T2 and T3, compared to T1. In contrast, one previous study showed smaller changes in sensitivity of TPOAb for predicting postpartum thyroid dysfunction (from 67 to $64 \%$ between 12 and 32 weeks, respectively) (13). Despite this, the authors still recommended for first, rather than third trimester TPOAb testing for prediction of PPTD (13).

This article is protected by copyright. All rights reserved. 
Inferences from the present study are limited by the sample size but are in keeping with the previous literature. It is possible some antibody levels could have already decreased prior to T1 testing, missing cases of thyroid autoantibody positivity and that false negative results were present among those patients who had TSH $>2.5$ and those with miscarriage. It is also possible that testing at a later time postpartum would pick up more cases of thyroid autoantibody positive women as it is known that the peak antibody titer in the postpartum phase can occur up to 6 months postpartum (25); therefore, median testing at 12 weeks postpartum could miss some women, whose levels peak later. Furthermore, with longer postpartum follow up, additional cases of PPTD may have been detected. The strengths of the study include its prospective nature, the measurements performed at each trimester and the presence of a postpartum measurement.

In conclusion, negative thyroid autoantibody results at $\mathrm{T} 2$ or $\mathrm{T} 3$ does not exclude autoimmune thyroid disease due to physiological decline in the positivity of thyroid autoantibodies with progression of pregnancy. Thyroid autoantibody testing at $\mathrm{T} 2$ and $\mathrm{T} 3$, compared to $\mathrm{T} 1$, also reduces the diagnostic accuracy for predicting PPTD. This indicates that testing at $\mathrm{T} 2$ and $\mathrm{T} 3$ to be of limited value. TPOAb and TGAb testing for investigation of thyroid dysfunction during pregnancy should occur with an awareness of this gestation dependent effect on the ability of the test to provide the desired information.

\section{REFERENCES}

1. Topliss, D.J., Eastman, C.J. (2004) 5: Diagnosis and management of hyperthyroidism and hypothyroidism. Med J Aust, 180,186-193.

2. Hollowell, J.G., Staehling, N.W., Flanders, W.D., et al. (2002) Serum TSH, T(4), and thyroid antibodies in the United States population (1988 to 1994): National Health and Nutrition Examination Survey (NHANES III). J Clin Endocrinol Metab, 87,489-499.

3. Stagnaro-Green, A., Roman, S.H., Cobin, R.H., et al. (1990) Detection of at-risk pregnancy by means of highly sensitive assays for thyroid autoantibodies. JAMA, 264,1422-1425.

This article is protected by copyright. All rights reserved. 
4. Glinoer D, R.M., Grün JP, Kinthaert J. (1994) Risk of subclinical hypothyroidism in pregnant women with asymptomatic autoimmune thyroid disorders. J Clin Endocrinol Metab., 79,197-204.

5. Lazarus, J.H. (2011) Thyroid function in pregnancy. Br Med Bull, 97,137-148.

6. Glinoer, D. (2004) The regulation of thyroid function during normal pregnancy: importance of the iodine nutrition status. Best Pract Res Clin Endocrinol Metab, 18,133-152.

7. Negro, R., Formoso, G., Mangieri, T., et al. (2006) Levothyroxine treatment in euthyroid pregnant women with autoimmune thyroid disease: effects on obstetrical complications. J Clin Endocrinol Metab, 91,2587-2591.

8. Negro, R., Schwartz, A., Gismondi, R., et al. (2010) Increased pregnancy loss rate in thyroid antibody negative women with TSH levels between 2.5 and 5.0 in the first trimester of pregnancy. $J$ Clin Endocrinol Metab, 95,E44-48.

9. Abalovich, M., Gutierrez, S., Alcaraz, G., et al. (2002) Overt and subclinical hypothyroidism complicating pregnancy. Thyroid, 12,63-68.

10. Haddow, J.E., Palomaki, G.E., Allan, W.C., et al. (1999) Maternal thyroid deficiency during pregnancy and subsequent neuropsychological development of the child. $N$ Engl J Med, 341,549-555.

11. Klein, R.Z., Sargent, J.D., Larsen, P.R., et al. (2001) Relation of severity of maternal hypothyroidism to cognitive development of offspring. J Med Screen, 8,18-20.

12. Smyth, P.P., Wijeyaratne, C.N., Kaluarachi, W.N., et al. (2005) Sequential studies on thyroid antibodies during pregnancy. Thyroid, 15,474-477.

13. Kuijpens, J.L., Pop, V.J., Vader, H.L., et al. (1998) Prediction of post partum thyroid dysfunction: can it be improved? Eur J Endocrinol, 139,36-43.

14. Parker, R.H., Beierwaltes, W.H. (1961) Thyroid antibodies during pregnancy and in the newborn. J Clin Endocrinol Metab, 21,792-798.

15. Stagnaro-Green, A., Abalovich, M., Alexander, E., et al. (2011) Guidelines of the American Thyroid Association for the diagnosis and management of thyroid disease during pregnancy and postpartum. Thyroid, 21,1081-1125.

16. De Groot, L., Abalovich, M., Alexander, E.K., et al. (2012) Management of thyroid dysfunction during pregnancy and postpartum: an Endocrine Society clinical practice guideline. J Clin

This article is protected by copyright. All rights reserved. 
Endocrinol Metab, 97,2543-2565.

17. Ekinci, E.I., Lu, Z.X., Sikaris, K., et al. (2013) Longitudinal assessment of thyroid function in pregnancy. Ann Clin Biochem, 50,595-602.

18. Gaberscek, S., Zaletel, K. (2011) Thyroid physiology and autoimmunity in pregnancy and after delivery. Expert Rev Clin Immunol, 7,697-706; quiz 707.

19. Balucan, F.S., Morshed, S.A., Davies, T.F. (2013) Thyroid autoantibodies in pregnancy: their role, regulation and clinical relevance. J Thyroid Res, 2013,182472.

20. Stagnaro-Green, A., Roman, S.H., Cobin, R.H., et al. (1992) A prospective study of lymphocyte-initiated immunosuppression in normal pregnancy: evidence of a T-cell etiology for postpartum thyroid dysfunction. J Clin Endocrinol Metab, 74,645-653.

21. Gonzalez, C., Hernando, M., Cava, F., et al. (2002) Biological variability of thyroid autoantibodies (anti-TPO and anti-Tg) in clinically and biochemically stable patients with autoimmune thyroid disease. J Clin Lab Anal, 16,37-39.

22. Muller, A.F., Drexhage, H.A., Berghout, A. (2001) Postpartum thyroiditis and autoimmune thyroiditis in women of childbearing age: recent insights and consequences for antenatal and postnatal care. Endocr Rev, 22,605-630.

23. Kent, G.N., Stuckey, B.G., Allen, J.R., et al. (1999) Postpartum thyroid dysfunction: clinical assessment and relationship to psychiatric affective morbidity. Clin Endocrinol (Oxf), 51,429-438.

24. Smallridge, R. (2000) Postpartum thyroid disease: a model of immunologic dysfunction. Clin Appl Immuonl Rev., 1,89-103.

25. Amino, N., Kuro, R., Tanizawa, O., et al. (1978) Changes of serum anti-thyroid antibodies during and after pregnancy in autoimmune thyroid diseases. Clin Exp Immunol, 31,30-37.

This article is protected by copyright. All rights reserved. 


\begin{tabular}{|c|c|c|c|c|c|}
\hline & \multirow[t]{2}{*}{ All } & \multicolumn{2}{|c|}{ TPOAb Status } & \multicolumn{2}{|c|}{ TGAb Status } \\
\hline & & Positive $^{a}$ & Negative & Positive $^{b}$ & Negative \\
\hline $\mathrm{n}$ & 140 & 13 & 127 & 15 & 125 \\
\hline Age (yr) & $31.3(28.4-34.8)$ & $34.6(29.4-39.4)^{*}$ & $31.3(28.3-34.5)^{*}$ & $33.3(29.4-36.5)$ & $31.3(28.2-34.5)$ \\
\hline Gravida & $2(2-4)$ & $3(2-4)$ & $2(1-4)$ & $2(2-3)$ & $2(1-4)$ \\
\hline Parity & $1(0-1)$ & $1(1-1)$ & $1(0-1)$ & $1(1-1)$ & $1(0-1)$ \\
\hline TSH $(\mathrm{mIU} / \mathrm{L})$ & $0.9(0.6,1.5)$ & $1.5(1.3,1.9)^{*}$ & $0.9(0.5,1.5)^{*}$ & $1.5(0.8,1.8)$ & $0.9(0.6,1.5)$ \\
\hline fT4 $(\mathrm{pmol} / \mathrm{L})$ & $15.1 \pm 2.8$ & $15.3 \pm 2.1$ & $15.1 \pm 2.9$ & $15.2 \pm 2.1$ & $15.1 \pm 2.9$ \\
\hline
\end{tabular}

TABLE 2. Outcomes of effects of time on positive TPOAb and TGAb status at trimester 1 .

\begin{tabular}{|c|c|c|}
\hline \multicolumn{3}{|c|}{ Effect of time on positive TPOAb status (compared to trimester 1 )* } \\
\hline & Odds Ratio (95\% CI) & $p$-value \\
\hline Trimester 2 & $0.04(0.002-0.8)$ & 0.03 \\
\hline Trimester 3 & $0.03(0.001-0.6)$ & 0.02 \\
\hline Postpartum & $2.2(0.3-15.8)$ & 0.4 \\
\hline \multicolumn{3}{|c|}{ Effect of time on positive TGAb status (compared to trimester 1 )*: } \\
\hline & Odds Ratio (95\% CI) & p-value \\
\hline Trimester 2 & $0.006(0.0001-0.3)$ & 0.01 \\
\hline Trimester 3 & $0.005(0.00007-0.4)$ & 0.02 \\
\hline Postpartum & $0.8(0.1-5.3)$ & 0.8 \\
\hline
\end{tabular}

*Analysis with random-effect model, adjusted for age and TSH.

This article is protected by copyright. All rights reserved. 


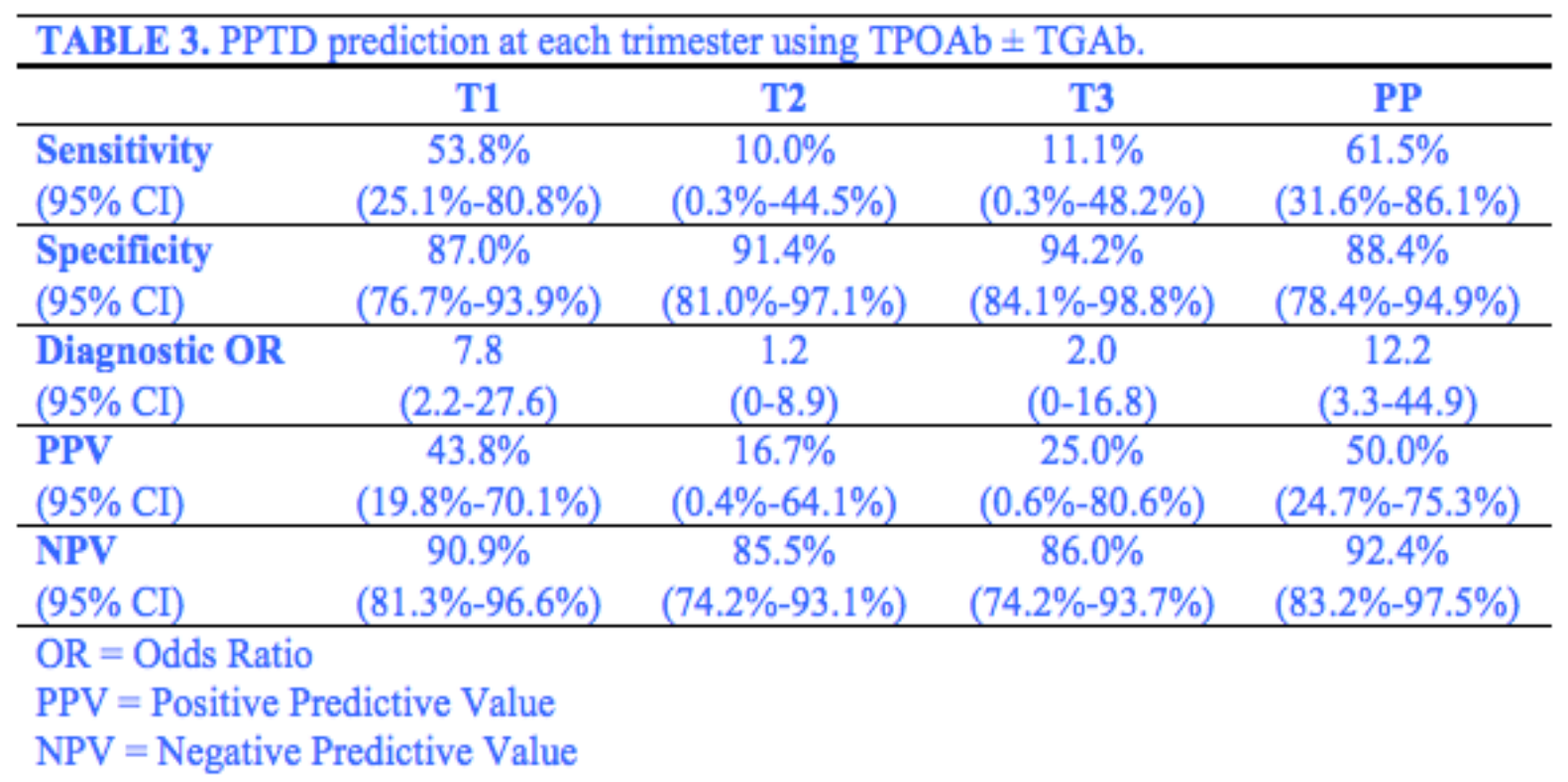

Figure 1. Scatterplots of thyroid autoantibody levels showing $25^{\text {th }}, 50^{\text {th }}$ and $75^{\text {th }}$ percentiles for (a) TPOAb and (b) TGAb levels. Trimester-1 (T1), trimester-2 (T2), trimester-3 (T3) and postpartum (PP).

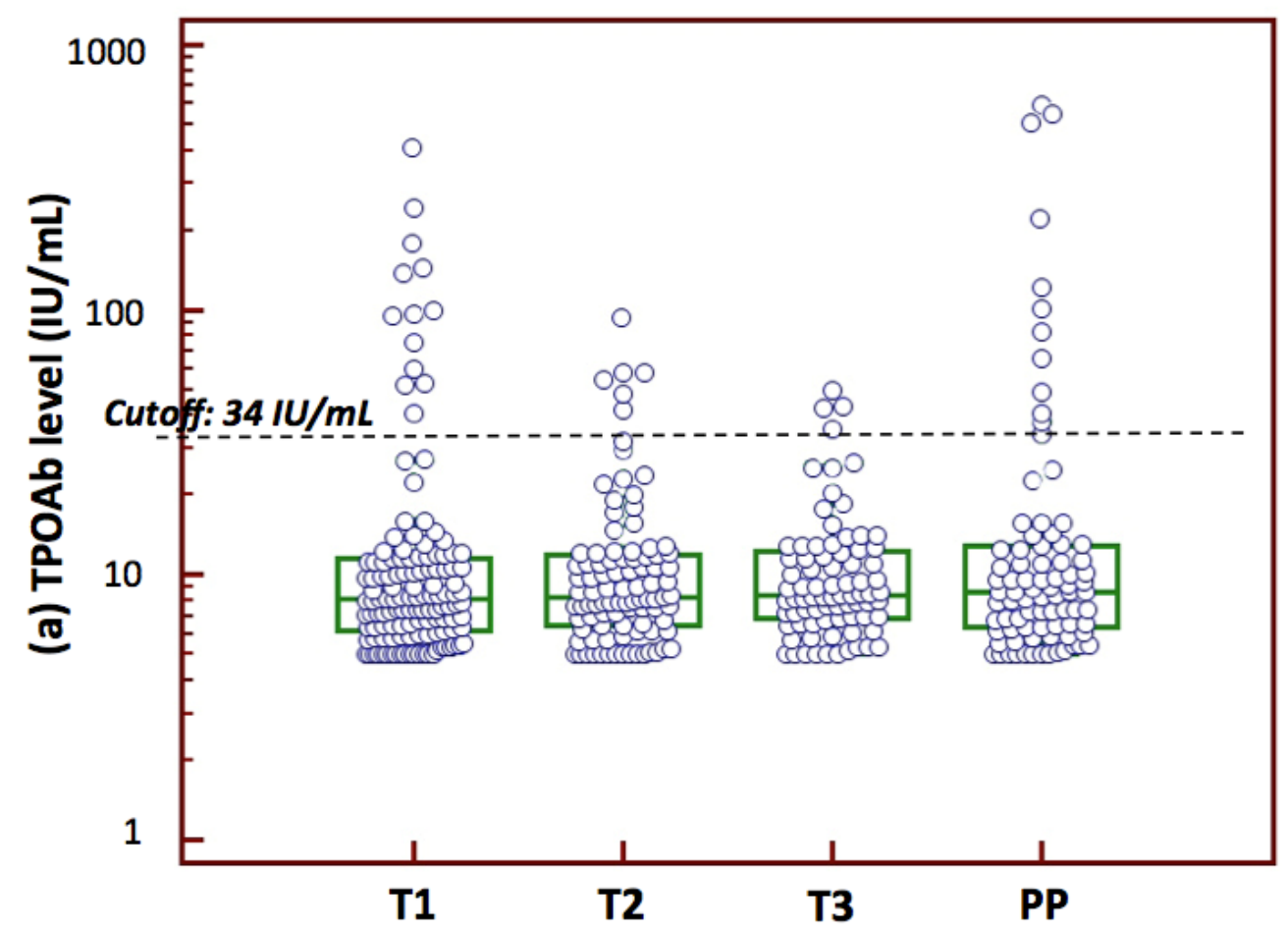

This article is protected by copyright. All rights reserved. 


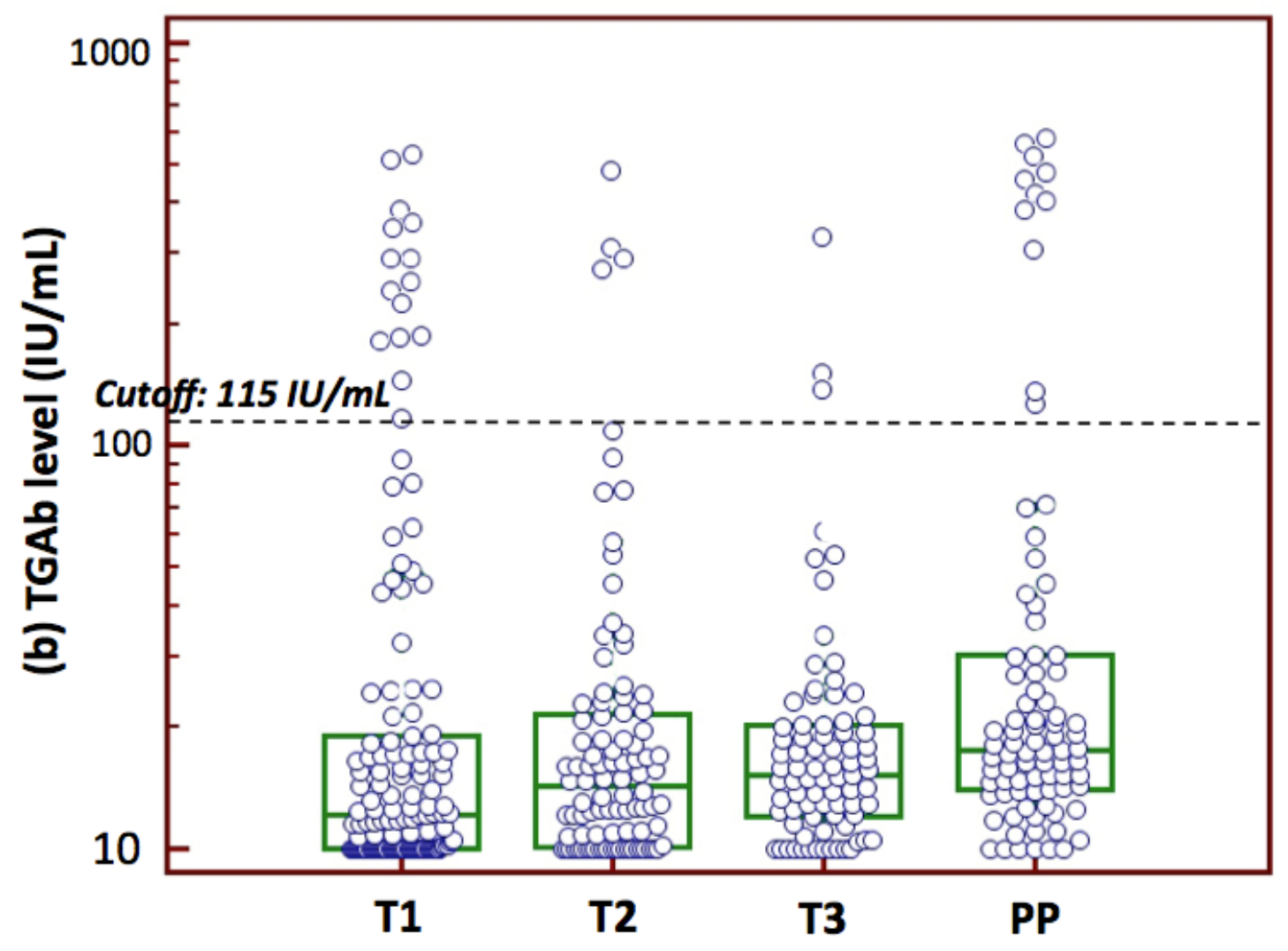

This article is protected by copyright. All rights reserved. 
Figure 2. Longitudinal changes in thyroid antibody levels of women with positive TPOAb (2a) and TGAb (2b) at T1, during pregnancy and postpartum.

Figure 2a.

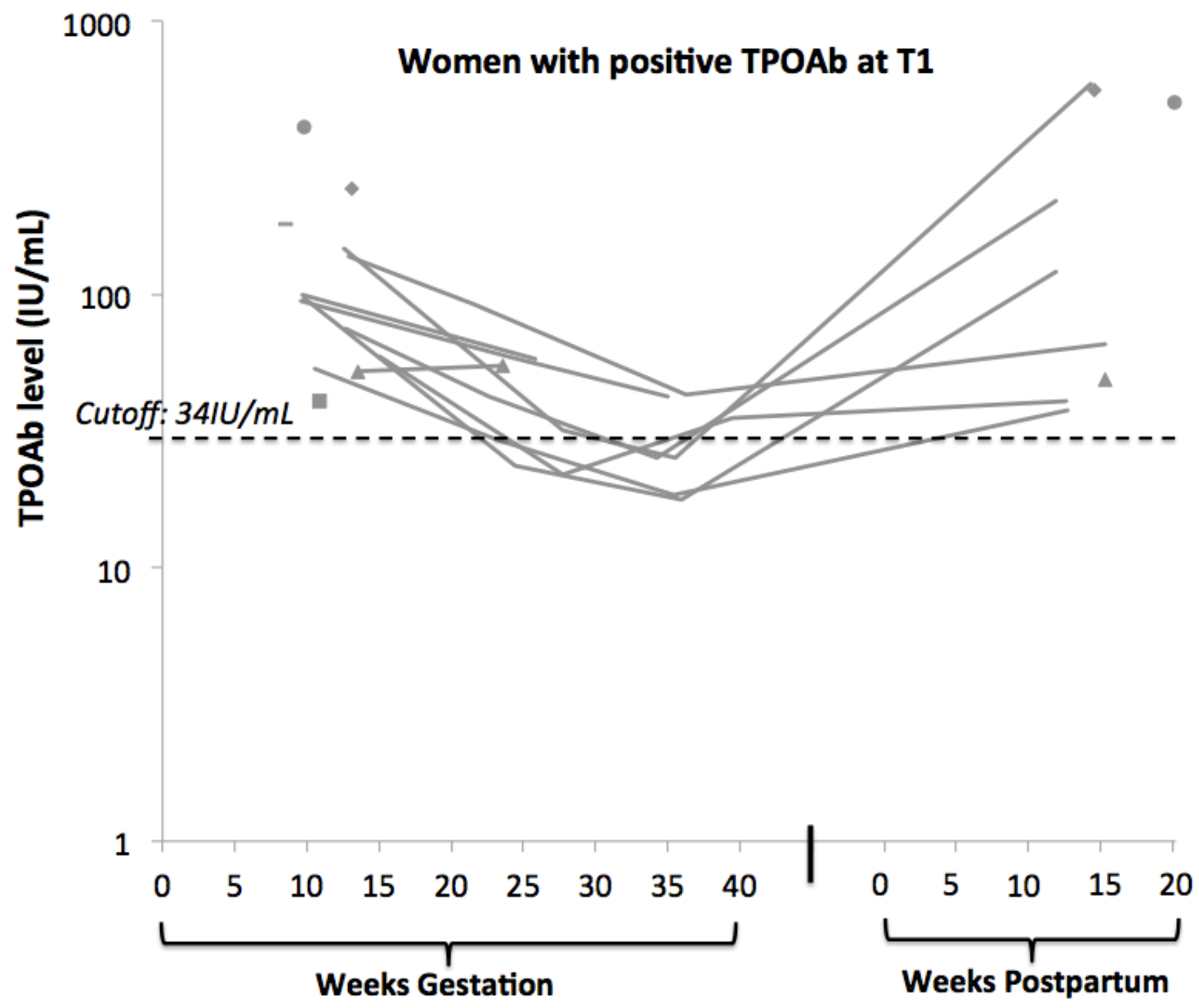

This article is protected by copyright. All rights reserved. 
Figure 2b:

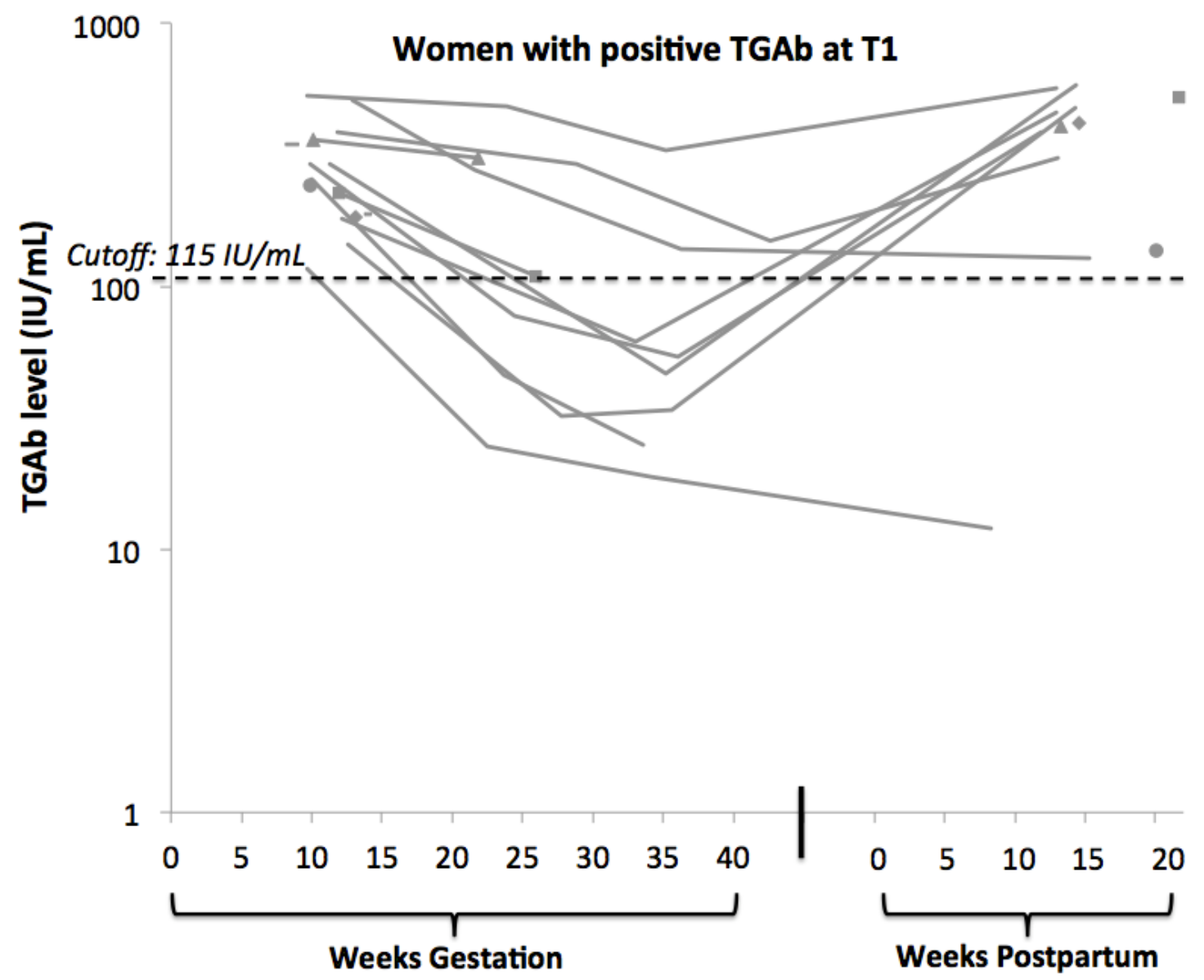

* Each symbol $(\bullet, \mathbf{\square}, \mathbf{\Delta}, \diamond$ or -$)$ indicates an individual patient with missing data at various timepoints. Continuous lines without symbol indicate individual patients without missing data.

This article is protected by copyright. All rights reserved. 